\title{
Pancreatic Tumors in Multiple Endocrine Neoplasia Type 1
}

$\mathrm{n}$ this issue, two excellent publications address the management of pancreatic neuroendocrine tumors in patients with Multiple Endocrine Neoplasia Type 1. The multifocal nature of the disease, the morbidity of removing the organ at risk, the morbidity of living without a pancreas, and the inconsistent malignant behavior of the tumors each affect the individual management decisions during the lifetime of a patient with MEN-1. The data in these manuscripts should help to guide us in these decisions.

Dr. Triponez and colleagues from the French Endocrine Tumor Study Group (GTE) present a summary of patients from their registry. ${ }^{1}$ They have chosen 65 patients who had nonfunctional pancreatic endocrine tumors $<2 \mathrm{~cm}$ in size by imaging studies. Their data show that the frequency of tumors increased between 1956 and 2003, consistent with the increasing sensitivity of imaging examinations. Fifteen of their 65 patients were managed by operation. In their registry, three patients are known to have died of tumor during follow-up. There was no difference in life expectancy of those patients with nonfunctioning pancreatic tumors compared to MEN-1 patients in their registry who did not have imageable pancreatic tumors.

These data give us an important note of caution regarding aggressive intervention for patients who might otherwise do well; however, the acknowledged weaknesses of the study may affect our interpretation. In particular, the short follow-up period of the group who did not have operation (mean $3.3 \mathrm{y}$ ) may affect these results. Also, the evaluation is limited to those patients who did not have a functional pancreatic tumor, and provides only small numbers for comparison among the groups. There was no consistent standard for imaging, particularly given the long time frame, which may affect the designation of a patient as being unaffected by tumors. Finally, as a non-randomized division between the operated and non-operated groups, we are left to wonder what the differences are that may have influenced the decision to operate. It is likely that differences between institutional approaches, individual surgeons, or unevaluated patient characteristics have affected the outcome. In spite of these limits, it is clear that most patients did very well, and there was no dramatic improvement in outcome for the small fraction of patients who did have operation.

Dr. Kouvaraki and colleagues have identified 55 patients from MD Anderson Cancer Center with pancreatic endocrine tumors and MEN-1, two-thirds of whom had functioning tumors. ${ }^{2}$ Thirty-eight of these patients underwent pancreatic operations. The rate of recurrent disease in the residual pancreas postoperatively was $20 \%$ at a median of 7.8 years, and the development of distant disease in those who presented without metastasis was $14 \%$ at a median of 2.7 years. Most importantly, they demonstrated an overall and metastasis-free survival improvement in their patients who underwent operation, compared to those with tumors who did not have operation.

This study also has some limits to its interpretation. These patients underwent a variety of operations for their disease, ranging from simple enucleation of tumors to total pancreatectomy. The criteria for selecting patients for operation, and the decision about which operation to perform, were not standardized and may have changed over time. The follow-up in this study is also short, at a median of 4.3 years. Finally, the follow-up program is not presented for us to understand how standardized this is in determining recurrence rates. The finding of survival improvement associated with intervention, even in a nonrandomized setting, is noteworthy.

Overall, these two important articles add to the data that we have to consider in our management of these patients and present differing views of the same disease (Fig. 1). The GTE study shows us that many patients with pancreatic endocrine tumors and MEN-1 do well and so we must be cautious in our interventions. However, the M.D. Anderson data indicate that there is some value to intervention in selected patients. For now, the significant outstanding issue in the management of pancreatic disease in MEN-1 remains the resolution of these views, including the proper surveillance regimen for best defining important disease, the optimal operative timing for prolongation of 
The Playing Field in MEN-1 Pancreas

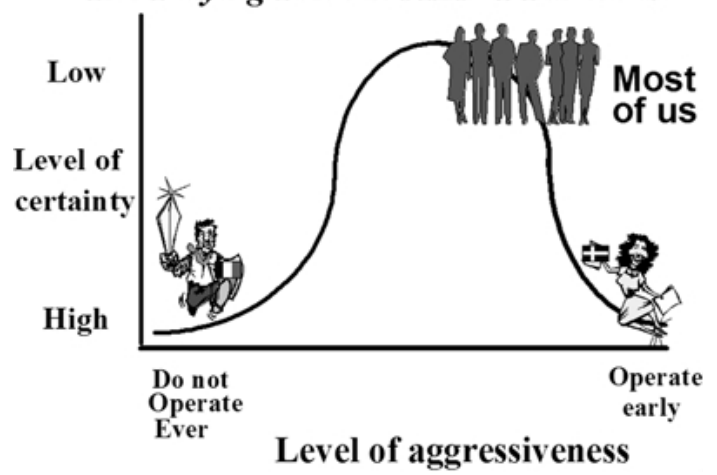

Figure 1. Decisions in MEN-1 pancreas intervention.

patient survival and quality of life, and the optimal operative intervention to achieve maximal oncologic benefit while preserving pancreatic endocrine function.

\section{REFERENCES}

1. Triponez $F$, Goudet $P$, Dosseh $D$, Cougard $P$, Bauters $C$, Murat A, Cadiot G, Niccoli-Sire P, Calender A, Proye CAG. Is surgery beneficial for MEN1 patients with small $(\leq 2 \mathrm{~cm})$, non functioning pancreaticoduodenal endocrine tumor? An analysis of 65 patients from the GTE. World J Surg (epub ahead of print, DOI: 10.1007/s00268-005-0354-9).

2. Kouvaraki MA, Shapiro SE, Cote G, Lee JE, Yao JC, Waguespack SG, Gagel RF, Evans DB, Perrier ND. Management of Pancreatic Endocrine Tumors in Multiple Endocrine Neoplasia Type 1. World J Surg (epub ahead of print, DOI: 10.I007/s00268-005-0360-y).

Gerard M. Doherty, MD Head, Section of General Surgery University of Michigan 2920 Taubman Center 1500 East Medical Center Drive Ann Arbor, MI 48109, USA e-mail: gerardd@umich.edu 